\title{
Sociodemographic factors associated with dietary supplement use in early pregnancy in a Swedish cohort
}

\author{
Linnea Bärebring ${ }^{1 *}$, Deirdre Mullally ${ }^{1}$, Anna Glantz ${ }^{2}$, Joy Elllis ${ }^{3}$, Lena Hulthén ${ }^{1}$, Åse Jagner ${ }^{2}$, \\ Maria Bullarbo ${ }^{4,5}$, Anna Winkvist ${ }^{1}$ and Hanna Augustin ${ }^{1}$ \\ ${ }^{1}$ Department of Internal Medicine and Clinical Nutrition, Sablgrenska Academy, University of Gothenburg, Box 459, 40530 \\ Gothenburg, Sweden \\ ${ }^{2}$ Department of Antenatal Care, Närbälsan, Primary Care, Kungsgatan 12, 41118 Gothenburg, Sweden. \\ ${ }^{3}$ Department of Antenatal Care, Närbälsan, Primary Care, Södra Bohuslän, MHV-enheten, Kungsgatan 12,411 19 \\ Gothenburg, Sweden \\ ${ }^{4}$ Södra Älvsborg Hospital, Brämbultsvägen 53, 501 82, Borås, Sweden \\ ${ }^{5}$ The Department of Obstetrics and Gynecology, Sablgrenska University Hospital, Östra, 416 85, Gothenburg, Sweden \\ (Submitted 5 June 2017 - Final revision received 4 September 2017 - Accepted 25 October 2017 - First published online 4 December 2017)
}

\section{Abstract}

Sociodemographic factors have been associated with dietary supplement use among pregnant women but few data exist in a Swedish population. This study aimed to identify factors associated with overall supplement use as well as use of folic acid, vitamin D and $n-3$ in early pregnancy. Women in the first trimester of pregnancy were included at registration to the antenatal care in 2013-2014 ( $n$ 2109). Information regarding supplement use as well as sociodemographic and anthropometric data were obtained from questionnaires and medical records. Multivariable logistic regression analysis was performed to determine the relationship between sociodemographic variables and supplement use. A total of $78 \%$ of the participants reported using at least one dietary supplement in the first trimester. Folic acid supplement use was reported by $74 \%$, vitamin D supplement use by $43 \%$ and $n-3$ supplement use by $<5 \%$. Use of any type of supplement in early pregnancy was related to gestational age, parity, birthplace, education and employment. Folic acid supplement use was related to gestational age, parity, birthplace, income, education and employment. Vitamin D supplement use was related to gestational age, birthplace and education. In conclusion, in the first trimester of pregnancy, folic acid supplements were used by three in four women, while vitamin D supplements were used by less than half of the women. The results of this study show a socioeconomic disparity between supplement users and non-users which may have a negative impact on the health of future generations.

Key words: Pregnancy nutrition: Dietary supplements: Folic acids: Vitamin D: $\boldsymbol{n}$-3 Fatty acids

The fetal origins hypothesis was proposed by Barker about three decades ago ${ }^{(1)}$ and since then research interest in maternal nutrition during pregnancy has increased significantly. The diet of women of childbearing age in Sweden has been shown to be insufficient in multiple micronutrients ${ }^{(2,3)}$. Also, there are indications that the dietary quality during pregnancy is lower than among non-pregnant women ${ }^{(4)}$. This is of concern due to increased nutritional requirements during pregnancy and the possible impact on the future health of the child.

Micronutrient supplementation during pregnancy has been examined in greater detail after the proof of the preventative function of folic acid supplementation against neural tube defects $^{(5)}$. The role of supplementation of other micronutrients vitamin $\mathrm{D}^{(6)}$ and $n-3$ fatty acids ${ }^{(7)}$ has also been examined with varying degrees of causal linkage to pregnancy outcomes, fetal or maternal health. In Sweden, the nutrition recommendations for supplement use during pregnancy are for women to take folic acid supplementation preconceptionally until gestational week 12 , and supplements of vitamin D and $n-3$ are recommended if dietary intakes of these nutrients are low ${ }^{(8)}$. Also, recommendations are to use iodine-fortified salt and iron supplements if iron deficient. In Sweden, women purchase supplements themselves, as they are not provided by the antenatal care.

Supplement use during pregnancy has been related to a variety of factors on both an individual and national level. In Europe, supplement use has been associated with sociodemographic factors such as level of education, maternal age, parity, tobacco use and $\mathrm{BMI}^{(9)}$. Studies which have examined folic acid supplement use have found higher rates of supplement use in countries which have specific recommendations for use periconceptually ${ }^{(10)}$. Data

Abbreviation: TEDDY, Environmental Determinants of Diabetes in the Young.

* Corresponding author: L. Bärebring, email linnea.barebring@gu.se 
from Denmark, Ireland and the Netherlands indicate that use of folic acid supplementation is associated with factors such age, parity, BMI, income, education and ethnicity ${ }^{(11-13)}$. n-3 Supplementation has long been popular in certain Nordic countries, particularly Norway and Iceland, and this also applies during pregnancy $^{(10,14)}$. There are however indications that $n-3$ supplement use is less common in Sweden ${ }^{(9)}$.

There is emerging evidence of difference in rates of supplement use during pregnancy, which may contribute further to the disparity between groups in terms of future health. The aim of this paper is to identify sociodemographic factors which may be related to dietary supplement use during early pregnancy.

\section{Methods}

\section{Subjects and study design}

The participants included in this investigation were recruited as part of the Swedish GraviD study. This is a population-based cohort study with the primary aim to investigate the association between maternal vitamin $\mathrm{D}$ status and hypertensive disorders during pregnancy ${ }^{(15,16)}$. Women who registered for antenatal care within the primary healthcare in Gothenburg, Södra Älvsborg and Södra Bohuslän during autumn 2013 and spring 2014 were invited to participate. This region of Sweden is representative of the whole country, in terms of the sociodemographic profile of the pregnant population ${ }^{(17)}$. The only exclusion criterion was pregnancy exceeding 16 gestational weeks at recruitment. In total, 2125 women were recruited and 2109 women were included in this study. The study was approved by the Regional Ethics Committee in Gothenburg and was conducted according to the Declaration of Helsinki. Written consent was obtained from all participants.

Questionnaires were completed by participants in the first and third trimesters (mean gestational weeks 11 and 34) at routine antenatal care visits. The questionnaires were administered by midwives and interpreters were available for assistance in order to overcome language barriers. The questionnaires included questions regarding diet, supplement use, lifestyle and personal information, including education, parity and country of birth. Medical records were obtained post-delivery and relevant information such as age, BMI, weight, employment status and tobacco use was obtained.

Information regarding income was not collected. Instead, mean incomes from different parts of Gothenburg were obtained from the Gothenburg City Council ${ }^{(18)}$, and mean incomes from other cities were obtained from Statistics Sweden ${ }^{(19)}$. Participants were thereafter categorised by the location of their antenatal care centre as a proxy for socioeconomic position of the region.

\section{Supplement use}

The questionnaires at both time points during pregnancy included a question regarding supplement use. This was asked as a yes/no question on vitamin supplement use with further details for those who answered yes. Although the question specified vitamins, the participant's answers included mineral and fatty acid supplements also. Multivitamin supplements were recorded as containing or not containing vitamin D and folic acid. When analysing vitamin $\mathrm{D}$ and folic acid supplement use, both single supplements and multivitamins were included. Fish oils were not present in any other supplement other than single supplements. Medical records were also reviewed to check for disparities in reported supplement use at registration to antenatal care. Fe supplementation was not considered, because the antenatal care actively screens all pregnant women for iron deficiency and prescribes supplementation when needed.

\section{Statistical analysis}

Statistical analysis was performed using IBM SPSS version 22. The sample size was based on power calculations that showed that a sample size of 2000 had $85 \%$ power to detect a doubled incidence of pregnancy-induced hypertension among women with vitamin D deficiency. Post boc power calculations showed that a sample size of 323 women was needed to determine the proportion of the population that used folic acid in early pregnancy (assuming 30\% use, 5\% precision and 5\% risk of type 1 error). Binary logistic regression was selected in order to determine the factors which were associated with the use of the supplements at registration to antenatal care as a dichotomous categorical variable. Age, BMI and gestational age were included as continuous variables. Parity, tobacco use and employment were dichotomized. Country of birth was divided into five categories; North Europe, America (North or South), Continental Europe, Asia and Africa. Education level was divided into three categories; primary level or less, secondary level or university level. Income of antenatal care area was divided into quartiles, where the first quartile represented the lowest income level. Significance level was set at $P<0.05$ in all analyses.

\section{Results}

\section{Participant characteristics}

A total of 2109 women were included in this study. Of these women, 1805 provided data on supplement use in both the first and third trimesters. Reasons for not providing data in late pregnancy included preterm delivery, pregnancy complications, pregnancy loss and terminated pregnancy. The sociodemographic characteristics of the GraviD cohort are presented in Table 1.

The mean age of the group was 31.4 (SD 4.9 ) years. The mean income of the group was approximately SEK 253000 yearly ( $\approx 25300$ euro). At registration to the antenatal care, mean BMI was $24.5 \mathrm{~kg} / \mathrm{m}^{2}$ and $4.3 \%$ reported current tobacco use. The majority of women were employed, born in North Europe and had a university level education. The mean gestational week at study inclusion was $10 \cdot 8$ weeks, when the first questionnaire was completed.

\section{Reported supplement use}

A total of $78 \%$ of the women used a supplement (any type) at inclusion in early pregnancy, and over half reported using a 
Table 1. Descriptive statistics of the 2109 pregnant women in the first trimester in the Swedish GraviD cohort

(Mean values and standard deviations; percentages and numbers)

\begin{tabular}{lcc}
\hline & Mean & SD \\
\hline Age (years) & $31 \cdot 4$ & $4 \cdot 9$ \\
BMI $\left(\mathrm{kg} / \mathrm{m}^{2}\right)$ & 24.5 & $4 \cdot 2$ \\
Mean income of area (SEK/year) & 253051 & 351470 \\
Gestational week at registration & $10 \cdot 8$ & $2 \cdot 0$ \\
\cline { 2 - 3 } & $\%$ & $n$ \\
\cline { 2 - 3 } & & \\
Tobacco use & 4.3 & 91 \\
Alcohol use & 1.4 & 28 \\
Pregnant with first child & $42 \cdot 3$ & 892 \\
North European origin & 75.6 & 1594 \\
Asian origin & 10.0 & 210 \\
Continental European origin & 6.9 & 146 \\
African origin & 5.6 & 118 \\
American origin & 1.9 & 41 \\
Unemployment & 18.5 & 386 \\
University level education & 59.8 & 1255 \\
\hline
\end{tabular}

Table 2. Reported supplement use in the pregnant Swedish GraviD cohort (Percentages and numbers)

\begin{tabular}{|c|c|c|c|c|}
\hline & \multicolumn{2}{|c|}{ Trimester 1 ( $n$ 2109) } & \multicolumn{2}{|c|}{ Trimester 3 ( $n 1805$} \\
\hline & $\%$ & $n$ & $\%$ & $n$ \\
\hline \multicolumn{5}{|c|}{ Any supplement use } \\
\hline Yes & 78 & 1642 & $59 \cdot 8$ & 1079 \\
\hline No & 22 & 463 & $40 \cdot 2$ & 726 \\
\hline \multicolumn{5}{|c|}{ Vitamin D supplement use } \\
\hline Yes & 43.3 & 913 & 39.4 & 710 \\
\hline No & $56 \cdot 7$ & 1195 & $60 \cdot 6$ & 1093 \\
\hline \multicolumn{5}{|c|}{ Folic acid supplement use } \\
\hline Yes & 74.4 & 1570 & 43.2 & 779 \\
\hline No & 25.5 & 538 & $56 \cdot 8$ & 1024 \\
\hline \multicolumn{5}{|c|}{$n-3$ Supplement use } \\
\hline Yes & 4.5 & 95 & 4.6 & 83 \\
\hline No & 95.5 & 2013 & 95.4 & 1720 \\
\hline
\end{tabular}

supplement in the third trimester (Table 2). At registration to antenatal care, $15.6 \%$ of the women took two supplements or more. Two supplements were reported by 274 women (13\%), three supplements by forty-one women ( $2 \%)$, four supplements by eleven women $(0.5 \%)$ and five women $(0.2 \%)$ reported taking five different supplements.

Approximately $74 \%$ reported taking folic acid in the first trimester (either as a single supplement or as part of a multivitamin) and $60 \%$ in the third trimester. A total of forty-one women reported that they had stopped taking folic acid at the time of registration. If these women are added, just over $78 \%$ took or had taken folic acid at the time of registration to antenatal care. Vitamin D supplementation was used by $43 \%$ at registration and $39 \%$ in the third trimester. $n-3$ Supplements were used by approximately $5 \%$ at both time points.

\section{Factors relating to supplement use in early pregnancy}

In multivariable logistic regression analysis, the factors relating to any supplement use at registration to antenatal care were gestational age, parity, birthplace, education level and employment (Table 3). Women who registered later for antenatal care, were parous, born in Continental Europe, Asia or Africa, had not attended university or were unemployed were less likely to be supplement users.

Folic acid supplement use in the first trimester of pregnancy was associated with gestational age, parity, income, birthplace, education and employment (Table 3). Women who registered later for antenatal care, were born in Continental Europe, Asia or Africa, had lower education and were unemployed, were less likely to take folic acid supplements, whereas women who were pregnant with their first child were more likely to use folic acid supplements. Also, women whose antenatal care facility was in an area within the second quartile of income were more likely to use supplements compared with women in the lowest quartile.

Factors relating to vitamin D supplement use at registration to antenatal care were gestational age, birthplace and education. Women who registered later for antenatal care, were born in Asia or Africa or had lower education were less likely to use vitamin D supplements (Table 3).

$n-3$ Supplement use at registration to antenatal care was not associated with any of the factors studied (data not shown).

\section{Discussion}

We found that $78 \%$ of women in the GraviD study reported using a dietary supplement in early pregnancy, and that $74 \%$ used folic acid at registration to antenatal care in gestational week $10 \cdot 8$. Vitamin D supplements were used by $43 \%$ and $n-3$ supplements by $5 \%$ of the women.

The total rate of supplement use among pregnant women in the GraviD study is similar but somewhat lower than the $81 \%$ reported in Norway ${ }^{(14)}$ and the $85 \%$ in Finland ${ }^{(20)}$. It is also slightly lower than what was reported during pregnancy in the Swedish Environmental Determinants of Diabetes in the Young (TEDDY) cohort in $2013^{(9)}$. However the rate of supplement use in the present study is higher than that reported in the All Babies in Southeast Sweden (ABIS) study conducted from 1997 to 1999 , where supplement use during pregnancy was reported by $56 \%$ of the women, retrospectively ${ }^{(21)}$. Use of supplements containing folic acid at registration to antenatal care was reported by $74 \%$ of pregnant women in this study. This number does not reflect pre-pregnancy supplement use. These data places Swedish folic acid use in the mid-range of supplement use, compared with other European countries ${ }^{(10)}$.

In the first trimester, $43 \%$ of pregnant women used a vitamin D supplement which is higher than the $27 \%$ reported in the ABIS study, carried out in 1997-1999 ${ }^{(21)}$. The higher proportion of vitamin D supplement use may reflect that the main focus of the GraviD project was to study the vitamin D status of the cohort, thus causing more women to use vitamin D supplementation. However, this is unlikely as the data were collected at study inclusion and most women took vitamin D as part of a multivitamin. A more likely explanation is that supplementary vitamin $\mathrm{D}$ intake has increased, as a reflection of an increased interest in the role of vitamin D in health and pregnancy and possibly a change in vitamin D content in multivitamins. This is supported 
Table 3. Multivariable logistic regression analysis of factors relating to supplement use in the first trimester $(\mathrm{T})$ in the pregnant Swedish GraviD cohort (Odds ratios and $95 \%$ confidence intervals)

\begin{tabular}{|c|c|c|c|c|c|c|c|c|c|}
\hline & \multicolumn{3}{|c|}{ Any supplement use } & \multicolumn{3}{|c|}{ Folic acid supplementation } & \multicolumn{3}{|c|}{ Vitamin D supplementation } \\
\hline & OR & $95 \% \mathrm{Cl}$ & $P$ & OR & $95 \% \mathrm{Cl}$ & $P$ & OR & $95 \% \mathrm{Cl}$ & $P$ \\
\hline Age $\mathrm{T} 1$ & 1.014 & $0.988,1.041$ & 0.305 & 1.014 & $0.988,1.040$ & 0.288 & 1.002 & $0.981,1.025$ & 0.831 \\
\hline BMI T1 & 0.987 & $0.960,1.014$ & 0.343 & 0.980 & $0.955,1.007$ & 0.141 & 1.002 & $0.979,1.025$ & 0.882 \\
\hline Gestational days $\mathrm{T} 1$ & $0.983^{*}$ & $0.975,0.990$ & $<0.001$ & $0.981^{*}$ & $0.973,0.988$ & $<0.001$ & $0.992^{*}$ & $0.986,0.999$ & 0.018 \\
\hline \multirow{2}{*}{\multicolumn{10}{|c|}{$\begin{array}{l}\text { Parity } \\
\text { Parous (Ref.) }\end{array}$}} \\
\hline & & & & & & & & & \\
\hline Nulliparous & $1.422^{*}$ & $1 \cdot 104,1 \cdot 830$ & 0.006 & $1.337^{\star}$ & $1.050,1 \cdot 703$ & 0.019 & 0.917 & $0.751,1.119$ & 0.394 \\
\hline \multirow{2}{*}{\multicolumn{10}{|c|}{$\begin{array}{l}\text { Origin } \\
\text { North Europe (Ref) }\end{array}$}} \\
\hline & & & & & & & & & \\
\hline America & 1.325 & $0.529,3.321$ & 0.548 & 1.660 & $0.658,4.188$ & 0.283 & 1.129 & $0.581,2.197$ & 0.720 \\
\hline Continental Europe & $0.521^{*}$ & $0.345,0.787$ & 0.002 & $0.526^{\star}$ & $0.352,0.787$ & 0.002 & 0.860 & $0.594,1.246$ & 0.425 \\
\hline Asia & $0.429^{*}$ & $0.298,0.618$ & $<0.001$ & $0.406^{\star}$ & $0.284,0.579$ & $<0.001$ & $0.585^{\star}$ & $0.412,0.831$ & 0.003 \\
\hline Africa & $0.423^{*}$ & $0.258,0.694$ & 0.001 & $0.357^{*}$ & $0.217,0.585$ & $<0.001$ & $0.575^{*}$ & $0.347,0.953$ & 0.032 \\
\hline \multirow{2}{*}{\multicolumn{10}{|c|}{$\begin{array}{c}\text { Income area of care } \\
\text { Quartile } 1 \text { (Ref.) }\end{array}$}} \\
\hline & & & & & & & & & \\
\hline Quartile 2 & 1.301 & $0.922,1.838$ & 0.135 & $1.548^{*}$ & $1 \cdot 105,2 \cdot 169$ & 0.011 & 0.902 & $0.675,1.204$ & 0.484 \\
\hline Quartile 3 & 1.060 & $0.744,1.513$ & 0.746 & 1.080 & $0.768,1.517$ & 0.659 & 0.939 & $0.699,1.262$ & 0.677 \\
\hline Quartile 4 & 0.728 & $0.508,1.043$ & 0.084 & 0.773 & $0.547,1.093$ & 0.145 & 0.887 & $0.657,1.197$ & 0.432 \\
\hline \multirow{2}{*}{\multicolumn{10}{|c|}{ Tobacco use T1 }} \\
\hline & & & & & & & & & \\
\hline Yes & 0.729 & $0.435,1.223$ & 0.231 & 0.848 & $0.505,1.423$ & 0.533 & 0.676 & $0.415,1.102$ & 0.116 \\
\hline \multicolumn{10}{|l|}{ Education level } \\
\hline \multicolumn{10}{|l|}{ University (Ref.) } \\
\hline Primary & $0.342^{*}$ & $0.219,0.533$ & $<0.001$ & $0.275^{\star}$ & $0.176,0.429$ & $<0.001$ & $0.510^{*}$ & $0.323,0.804$ & 0.004 \\
\hline Secondary & $0.590^{*}$ & $0.451,0.772$ & $<0.001$ & $0.616^{*}$ & $0.475,0.798$ & $<0.001$ & $0.729^{*}$ & $0.586,0.906$ & 0.004 \\
\hline \multicolumn{10}{|c|}{ Employment } \\
\hline Employment/parenta & & & & & & & & & \\
\hline Unemployed & $0.674^{*}$ & $0.495,0.917$ & 0.012 & $0.727^{\star}$ & $0.537,0.986$ & 0.040 & 0.792 & $0.599,1.045$ & 0.099 \\
\hline
\end{tabular}

Ref., referent values.

${ }^{*} P<0.05$. 
by the 2013 TEDDY study, where $48 \%$ of Swedish pregnant women reported using a vitamin D-containing supplement during pregnancy which is comparable to our findings ${ }^{(9)}$.

$n-3$ Supplement use in early pregnancy was reported by $4.5 \%$ of the women in the GraviD cohort. This is similar to the $7 \cdot 0 \%$ reported among Swedish women by the TEDDY study ${ }^{(9)}$. These figures are significantly lower than the $59 \%$ reported in Norway $^{(14)}$ or the $95 \%$ among Icelandic women ${ }^{(10)}$. These results show that although the Nordic countries have many similar dietary habits, fish oil supplement use is not uniform between countries.

No supplement use in early pregnancy was associated with being parous, registering later for antenatal care, being of continental European, Asian or African origin, having lower education level and being unemployed. These data are supported by findings from the TEDDY study where nulliparity and higher education were associated with supplement use during pregnancy in Sweden ${ }^{(9)}$. However, the TEDDY study did not present any data on ethnicity or employment in their Swedish cohort. It has been suggested that women's inclination to take tablets during pregnancy may vary between countries ${ }^{(22)}$. Lower availability or use of supplements in general in other countries may mean that supplement use during pregnancy is not a common practice. This practice may be preserved upon relocation to Sweden. French data suggest that preconception folic acid use is less common among women born in Africa, in line with our findings ${ }^{(23)}$. To our knowledge, this has previously never been investigated in the Swedish pregnant population. It is possible that women with lower socioeconomic position are less likely to use supplements due to the cost, as pregnant women in Sweden are not provided with supplements free of charge. Having an immigrant background in Sweden means that you are more likely to have a lower socioeconomic position, reflected by lower education, higher unemployment rates and lower income ${ }^{(24)}$. However, as birthplace, lower education and unemployment were all related to less supplement use in the same regression model, these aspects of poorer socioeconomic position all seem to be of importance for supplement use, independently. Similarly to any supplement use, folic acid supplement use was associated with gestational age, nulliparity, country of birth, higher education, employment and income. The increased likelihood of supplement use among women who were employed or whose antenatal care facility was located in the second quartile of income could be a reflection of socioeconomic determinants of folic acid use. As income was defined as the income of the area of the antenatal care clinic, it is also possible that the different clinics put different emphasis on folic acid use. Gestational age at registration for antenatal care, and inclusion in the study, was negatively associated with folic acid supplement use. This could be a reflection of the recommendation that folic acid use should be used until gestational week 12, and women who had surpassed this week might already have discontinued folic acid use. It is also possible that women who register later for antenatal care are less inclined to follow the recommendation regarding use of folic acid supplements.

Women born in Asia, continental Europe or Africa had a reduced likelihood of folic acid use in the first trimester compared with women born in northern Europe, which is similar to the findings of overall supplement use in this study. Vitamin D supplement use during pregnancy was seen to be mainly influenced by socioeconomic factors and birthplace in the GraviD study. Non-use was associated with later registration for antenatal care, Asian or African origin, unemployment and lower education. As these are also risk groups for vitamin D deficiency, this might indicate that the women who need vitamin D supplementation the most are the least likely to use it. $n$-3 supplement use was not related to any sociodemographic variable in this study. This might be a reflection of insufficient statistical power as the number of $n-3$ supplements users was low.

The present study is one of few studies examining factors relating to overall and specific supplement use among pregnant women in Sweden. One of the strengths of the GraviD study is the relatively large sample size. The cohort is also populationbased, and has high participation rates among women born in other countries. Also, supplement use was both reported in questionnaires and retrieved from medical charts. Some limitations of the present study include the fact that information was not gathered on supplement use before pregnancy which means that compliance to the recommendation of preconceptional use of folic acid could not be fully investigated. Also, not including frequency and dose of supplementation may have led to an overestimation of supplement use in this cohort. Fe supplementation was not included in this paper, though most multivitamin supplements did contain smaller amounts. Fe supplementation was excluded because of the antenatal care's programme to find and treat women with Fe deficiency. The fact that Fe supplementation is, when needed, prescribed from the healthcare sets it apart from other supplements. The income levels in the present study were captured by a proxy variable based on the location of the antenatal care centre attended. Also, the proportion of those with a university level education was slightly higher than the national average. Lastly, only 1805 of the 2109 women provided data also on supplement use in the third trimester of pregnancy. However, excluding the women with missing data from the third trimester did not alter the frequency of supplement use in the first trimester.

In conclusion, $78 \%$ of the women in this study took a dietary supplement when registering for antenatal care. Folic acid supplementation was reported by $74 \%$, vitamin D supplementation by $43 \%$, and $n-3$ Supplements by $<5 \%$. There were socioeconomic disparities in the use of dietary supplements in early pregnancy. Differences in nutritional behaviour during pregnancy may predispose babies to further inequalities. Therefore, vulnerable socioeconomic groups might benefit from reduced costs of supplements or increased support from antenatal care to comply with recommended supplement use during pregnancy.

\section{Acknowledgements}

The authors would like to thank the women who participated in the study, as well as the midwives and nurses whose contributions were pivotal in the realisation of the study. 
This research was funded by the Swedish Research Council for Health, Working Life and Welfare (H. A., grant no. 20120793) and Regional Research and Development grants (M. B., grant nos VGFOUREG-388201, VGFOUREG-229331). The funders had no role in the design, analysis or writing of this article.

L. B. and D. M. contributed with formulating the research question, data collection, data analysis and writing this article. A. G., J. E., L. H., A. J., M. B. and A. W. contributed with study design, formulating the research question and writing this article. H. A. is the principal investigator and contributed with study design, formulating the research question, data collection and writing this article.

None of the authors has any conflicts of interest to declare.

\section{References}

1. Barker DJ (1990) The fetal and infant origins of adult disease. BMJ 301, 1111

2. Amcoff E, Edberg A, Enghardt Barbieri H, et al. (2010-11) Riksmaten - vuxna 2010-11. Livsmedels- och näringsintag bland vuxna i Sverige. Uppsala: National Food Agency.

3. Becker W, Lindroos AK, Nälsén C, et al. (2016) Dietary habits, nutrient intake and biomarkers for folate, vitamin $\mathrm{D}$, iodine and iron status among women of childbearing age in Sweden. Ups J Med Sci 121, 271-275.

4. Lundqvist A, Johansson I, Wennberg AL, et al. (2014) Reported dietary intake in early pregnant compared to non-pregnant women - a cross-sectional study. BMC Pregnancy Childbirth 14, 373.

5. Medical Research Council Vitamin Study Research Group (1991) Prevention of neural tube defects: results of the Medical Research Council Vitamin Study. Lancet 338, 131-137.

6. Thorne-Lyman A \& Fawzi WW (2012) Vitamin D during pregnancy and maternal, neonatal and infant health outcomes: a systematic review and meta-analysis. Paediatr Perinat Epidemiol 26, 75-90.

7. Larqué E, Gil-Sánchez A, Prieto-Sánchez MT, et al. (2012) Omega 3 fatty acids, gestation and pregnancy outcomes. Br J Nutr 107, S77-S84.

8. The National Food Agency (2008) Advice About Food for You Who Are Pregnant. Uppsala: National Food Agency.

9. Aronsson CA, Vehik K, Yang J, et al. (2013) Use of dietary supplements in pregnant women in relation to sociodemographic factors - a report from the Environmental Determinants of Diabetes in the Young (TEDDY) study. Public Health Nutr 16, 1390-1402.

10. Oliver EM, Grimshaw KEC, Schoemaker AA, et al. (2014) Dietary habits and supplement use in relation to national pregnancy recommendations: data from the EuroPrevall Birth Cohort. Matern Child Health J 18, 2408-2425.
11. Cueto HT, Riis AH, Hatch EE, et al. (2012) Predictors of preconceptional folic acid or multivitamin supplement use: a cross-sectional study of Danish pregnancy planners. Clin Epidemiol 4, 259-265.

12. McKeating A, Farren M, Cawley S, et al. (2015) Maternal folic acid supplementation trends 2009-2013. Acta Obstet Gynecol Scand 94, 727-733.

13. Manniën J, De Jonge A, Cornel MC, et al. (2013) Factors associated with not using folic acid supplements preconceptionally. Public Health Nutr 17, 2344-2350.

14. Haugen M, Brantsæter AL, Alexander J, et al. (2008) Dietary supplements contribute substantially to the total nutrient intake in pregnant Norwegian women. Ann Nutr Metab 52, 272-280.

15. Bärebring L, Bullarbo M, Glantz A, et al. (2016) Preeclampsia and blood pressure trajectory during pregnancy in relation to vitamin D status. PLOS ONE 11, e0152198.

16. Bärebring L, Schoenmakers I, Glantz A, et al. (2016) Vitamin D status during pregnancy in a multi-ethnic population-representative Swedish cohort. Nutrients $\mathbf{8}, 655$.

17. Socialstyrelsen (2015) Pregnancies, Deliveries and Newborn Infants: The Swedish Medical Birth Register 1973-2014, no. 1400-3511]. Stockholm: Official Statistics of Sweden.

18. Statistik och Analys stadsledningskontoret Göteborgs Stad (2013) Statistik Göteborg (Statistics Gothenburg). http:// statistikdatabas.goteborg.se/pxweb/sv/Statistik\%20G\%C3\% B6teborg/Statistik\%20G\%C3\%B6teborg_Prim\%C3\%A4romr\% C3\%A5den__Inkomst\%20och\%20utbildning__Inkomster/10_ Inkomster_PRI.px/?rxid=0381a857-369f-4522-8e3c-1859f4c18178 (accessed February 2016).

19. Statistics Sweden (2014) Inkomst av tjänst (antal personer, medelvärden och totalsumma) efter region, kön, ålder och inkomstklass. År 2000-2014 (Income by region, gender, age and income class. Years 2000-2014). http://www.statistikdatabasen. scb.se/pxweb/sv/ssd/START_HE_HE0110_HE0110A/InkAvT janst/?rxid=fd771a78-3d45-4624-b631-0e0142f385ce (accessed February 2016).

20. Arkkola T, Uusitalo U, Pietikäinen M, et al. (2006) Dietary intake and use of dietary supplements in relation to demographic variables among pregnant Finnish women. Br J Nutr 96, 913-920.

21. Brekke HK \& Ludvigsson J (2007) Vitamin D supplementation and diabetes-related autoimmunity in the ABIS study. Pediatr Diabetes 8, 11-14.

22. Nagata JM, Gatti LR \& Barg FK (2012) Social determinants of iron supplementation among women of reproductive age: a systematic review of qualitative data. Matern Child Nutr 8, 1-18.

23. Tort J, Lelong N, Prunet C, et al. (2013) Maternal and health care determinants of preconceptional use of folic acid supplementation in France: results from the 2010 National Perinatal Survey. BJOG 120, 1661-1667.

24. Taguma M, Kim M, Brink S, et al. (2010) OECD Reviews of Migrant Education - Sweden. Paris: OECD. 